\title{
Static Vs Dynamic Connectivity: How Landscape Changes Affect Connectivity Predictions in the Iberian Peninsula
}

\author{
Teresa Goicolea ( $\nabla$ t.goicolea@gmail.com ) \\ Universidad Politécnica de Madrid https://orcid.org/0000-0002-4069-6001 \\ María Cruz Mateo-Sánchez \\ Universidad Politecnica de Madrid
}

\section{Research Article}

Keywords: spatio-temporal connectivity, time-varying connectivity, habitat availability metrics, landscape dynamics, biodiversity conservation, global change

Posted Date: November 10th, 2021

DOl: https://doi.org/10.21203/rs.3.rs-1044789/v1

License: (c) (i) This work is licensed under a Creative Commons Attribution 4.0 International License.

Read Full License 


\section{Abstract}

Context Climate and land-use changes affect species ranges and movements. However, these changes are normally overlooked in connectivity studies, and this could have adverse consequences in the definition of effective management measures.

Objectives We evaluated two ways to incorporate this dynamism: (i) by acknowledging that connectivity is a fluctuating phenomenon (i.e., time-varying connectivity) and therefore, procuring long-term conservation measures; and (ii) by enhancing species movements to their future ranges (i.e., spatiotemporal connectivity). We further compared these dynamic approaches with traditional static connectivity methods.

Methods We compared the overall connectivity values and the prioritization of critical habitat patches according to the dynamic and static approaches. This comparison research was conducted for species associated with broadleaf forests of the different ecoregions of the Iberian Peninsula. We considered species habitat preferences for moving and a wide range of dispersal abilities to assess functional connectivity without focusing on a single species.

Results Static approaches generated varying overall connectivity values and priority patches depending on the time snapshot considered and different from those generated by dynamic approaches. The two dynamic connectivity approaches resulted in very similar priority conservation patches, indicating their potential to guide endurable conservation measures that enhance connectivity between contemporaneous habitat patches at multiple time snapshots but also species range shifts in time.

Conclusions Connectivity is affected by landscape changes, and only dynamic approaches can overcome the issues associated with these changes and provide valuable information to guide improved and endurable measures in changing landscapes.

\section{Introduction}

Landscapes change due to climate (Mora et al. 2013), and land-use variations (Song et al. 2018), and the natural dynamism of habitats. These changes may affect vegetation distribution and structure (Root et al. 2003; Beltrán et al. 2014), water availability (Taylor et al. 2013; Bishop-Taylor et al. 2018a), and other factors affecting species habitats. Changes in habitat distribution, amount, and quality may result in species' ranges shifts (among other responses such as phenological, behavioral, or genetic adaptation) to ensure species perdurance (Parmesan and Yohe 2003; Chen et al. 2011; Davidson et al. 2020; RománPalacios and Wiens 2020). However, habitat fragmentation could complicate and even jeopardize species colonization of new areas with suitable habitat (Collinge 1996; Honnay et al. 2002). The enhancement of habitat connectivity is considered a key strategy to mitigate the detrimental effects of habitat fragmentation, as it eases the flow of resources, species, genes, and ecological processes through the landscape (Correa Ayram et al. 2015; Keeley et al. 2018a). 
Changes in the spatial configuration, amount, and quality of species habitats, as well as the matrix inbetween habitat patches, may alter the flow of species and ecological processes, involving changes in connectivity over time. Therefore, connectivity is increasingly recognized as a time-varying phenomenon (Keeley et al. 2018b; Zeller et al. 2020). Nevertheless, connectivity has traditionally been studied through a static and immutable approach, which identifies landscape areas that promote the exchange of individuals and processes between contemporaneous habitat patches (Nuñez et al. 2013; Albert et al. 2017; Costanza et al. 2020). Connectivity changes over time have been only recently incorporated into connectivity studies by analyzing landscapes at a unique future snapshot or multiple time snapshots (Saura et al. 2011; Beltrán et al. 2014; Bishop-Taylor et al. 2018b; Jennings et al. 2020). These studies have corroborated that connectivity is a dynamic phenomenon and does not remain constant over time. They usually identify stable areas (i.e., habitat patches or corridors) that persist with suitable conditions for the focal species over time as targets for conservation measures (the so-called climate refugia in the case of climate change). Considering connectivity as a time-varying phenomenon is important to adequately guide long-term management measures that enhance functional connectivity over a broad period of time.

So far, dynamic connectivity studies only considered changes in connectivity over time (i.e., time-varying connectivity) but rarely considered whether landscape features would allow the shift of species or processes at a specific snapshot to their future suitable ranges (Littlefield et al. 2017; Martensen et al. 2017; Carroll et al. 2018; Gray et al. 2020; Huang et al. 2020; Zhao et al. 2021). However, the distribution of species habitat and the surrounding landscape change, and the pattern of these changes may become a key factor to enable organisms to reach their future potential distribution areas. Thus, considering the connectivity between the different snapshots (i.e., spatio-temporal connectivity) might provide a step forward to allow more realistic, robust, and ecologically meaningful connectivity assessments. The scarce work reported on this topic was also greatly simplified as most studies did not account for particularities of the dynamism, the focal species, and/or the landscape. For instance, most of them did not consider network directionality (Acevedo et al. 2015), i.e., flow can only occur from a past to a future snapshot, and not in the opposite direction. Another usual simplification was connecting climate refugia. This implied considering climate as the only important factor in species habitat selection, although other variables as anthropic pressure or land cover are known to be of most importance in habitat selection (Mateo Sánchez et al. 2014; Gastón et al. 2019; Banfield et al. 2020). Other spatio-temporal connectivity assessments studied the connections between static protected areas; however, these areas might change (Elsen et al. 2020) and stop being suitable for species in the future. Furthermore, very few functional connectivity studies have accounted for the dynamism of landscapes and, at the same time, for species dispersal abilities and habitat preferences for movement (Keeley et al. 2018b; Zeller et al. 2020). In fact, most dynamic studies have disregarded the state and changes in the landscape matrix and how it impacts species movements, that is, the landscape resistance (Spear et al. 2010; Zeller et al. 2012; MateoSánchez et al. 2015; Keeley et al. 2016). Integrating all these particularities would lead to an improved evaluation of spatio-temporal connectivity to comprehensively guide sound and feasible decisions for landscape management. 
Importantly, and due to the limited resources allocated to conservation actions, it is essential to identify and focus efforts and means on the most critical landscape areas. A few time-varying and spatiotemporal connectivity studies prioritized conservation areas (Albert et al. 2017; Bishop-Taylor et al. 2018b; Jennings et al. 2020; Conlisk et al. 2021; Zhao et al. 2021). These studies mainly accounted for the perdurance in time of quality areas or their spatial probability of connection (i.e., effective distance). However, apart from these characteristics, it is important to regard the irreplaceability of the areas. In other words, consider whether alternative landscape elements could, at least partially, supplant the contribution to the overall connectivity of the considered area in case it gets lost or degraded. Some graph-based (Urban et al. 2009) habitat availability metrics have been proposed to include irreplaceability in connectivity prioritization: the Probability of Connectivity (PC) (Saura and Pascual-Hortal 2007; Saura and Rubio 2010), and the Equivalent Connected Area (ECA) (Saura et al. 2011; Santini et al. 2016), which have been proven to be good indicators to monitor landscape changes (Bishop-Taylor et al. 2018a; Poli et al. 2019; Keeley et al. 2021). Martensen et al. 2017 adapted these metrics to be applied in spatio-temporal connectivity studies. These newly customized metrics can be used to calculate the reachable habitat through spatial and temporal connections. Yet, these metrics have only been applied to calculate overall dynamic connectivity levels (Huang et al. 2020) and patches Betweenness Centrality (Zhao et al. 2021). These studies do not calculate the individual irreplaceability of landscape elements to identify key areas that promote species movements under changing conditions.

Our goal here was to gain insights about how to incorporate the dynamism in connectivity studies to (a) calculate overall connectivity and (b) prioritize individual habitat patches by their contribution to overall connectivity. For that purpose, we performed a quantitative and qualitative comparative study on the implications of three connectivity approaches: (i) static, (ii) time-varying, and (iii) spatio-temporal. Specifically, we compared the overall connectivity, and priority patches obtained from these three approaches in the time period from 1990 (initial snapshot, t1) to 2018 (final snapshot, t2). To do so, we focused on broadleaf forests along the different ecoregions of the Iberian Peninsula. We followed a functional approach (i.e., a resistance-based approach that accounts for species habitat selection) (Tischendorf and Fahrig 2000) in a multispecies framework (i.e., we considered a wide range of dispersal distances to capture the variable dispersal abilities of the species associated with the focal habitat). Therefore, this study also evaluated the change in connectivity for (a) species with different vagility (Costanza et al. 2020) and (b) among ecoregions with different ecological and climatic characteristics and trends (Olson et al. 2001). As far as we are aware, this study is the first analysis of time-varying and spatio-temporal connectivity that includes the mentioned particularities of the dynamism, the focal species, and the landscape matrix and prioritizes habitat patches by their contribution (or importance) and irreplaceability. Moreover, this work contributes to a more comprehensive understanding of the implications of incorporating dynamism in connectivity studies to guide more efficient and informed conservation planning.

\section{Materials And Methods}




\subsection{STUDY AREA, ECOREGIONS, AND SPECIES}

This study was carried out on the Iberian Peninsula (Fig. 1), South-Western Europe, an area especially affected by landscape changes (Loarie et al. 2009; Song et al. 2018). It has an extension of $581,000 \mathrm{~km}^{2}$ and comprises two countries, Spain and Portugal. It is a heterogeneous area that encompasses very different ecological characteristics. Therefore, we divided the study area into biogeographic units or ecoregions with similar species, dynamics, and environmental conditions (Fig. 1, Table 1). This division was given by the terrestrial ecoregions of the world delineated by Olson et al. 2001. We hypothesized that species would only roam between habitat patches with similar ecological conditions. Therefore, we only considered the connections among habitat patches (i.e., broadleaf forests) belonging to the same ecoregion.

Table 1

Ecoregions of the Iberian Peninsula

\begin{tabular}{|lll|}
\hline ID & Ecoregion name & Area (km2) \\
\hline 6 & Cantabrian mixed forests & 76366.89 \\
\hline 8 & Iberian conifer forests & 34439.47 \\
\hline 9 & Iberian sclerophyllous and semi-deciduous forests & 297966.58 \\
\hline 15 & Northeastern Spain and Southern France Mediterranean forests & 27915.62 \\
\hline 16 & Northwest Iberian montane forests & 57449.96 \\
\hline 33 & Pyrenees conifer and mixed forests & 13419.41 \\
\hline 19 & Southeastern Iberian shrubs and woodlands & 2710.95 \\
\hline 21 & Southwest Iberian Mediterranean sclerophyllous and mixed forests & 70568.26 \\
\hline
\end{tabular}

The diversity of focal species (i.e., broadleaf forest specialists) was represented by a wide range of median dispersal distances: $1 \mathrm{~km}, 2 \mathrm{~km}, 5 \mathrm{~km}, 10 \mathrm{~km}, 30 \mathrm{~km}$, and $50 \mathrm{~km}$. These distances condition species arrival to destination habitat patches and capture most dispersal abilities of terrestrial animals (Sutherland et al. 2000; Saura et al. 2011, 2017; Santini et al. 2013).

\subsection{METHODS}

We compared three different approaches to study connectivity: (i) static; (ii) time-varying; and (iii) spatiotemporal. The static approach (i.e., considering connections between contemporaneous habitat patches at a single time snapshot) was followed twice independently, one at an initial time snapshot t1 (1990) and another one at a final snapshot t2 (2018). These two static runs were denoted by s1 and s2 respectively. The time-varying approach (denoted by $\mathrm{tv}$ ) considered together both time snapshots ( $\mathrm{t} 1$ and 
t2) just by comparing or adding the results of $s 1$ and s2. This approach informed about how connectivity changed over time. Lastly, the spatio-temporal approach (denoted by st) studied the connection of habitat patches at $\mathrm{t} 1$ with those at $\mathrm{t} 2$. All analyses were developed separately for each ecoregion and dispersal distance.

The methods of this article are structured as follows (Fig. 2): Firstly, we defined the study scenarios (i.e., the combination of network and matrix characteristics) in section 2.3. To do so, we (a) identified the habitat patches (section 2.3.1), (b) calculated the resistance surface (section 2.3.2), (c) determined the connections between patches and its spatial probability of connection (section 2.3.3), and (d) calculated the temporal probability of the connections in the spatio-temporal scenario (section 2.3.4). Secondly, we carried out the connectivity analyses (section 2.4) to measure (a) the overall connectivity (section 2.4.1) and (b) the individual node importance to ultimately identify priority conservation patches (section 2.4.2). Lastly, we compared the connectivity results of each approach (static, time-varying, or a spatio-temporal), ecoregion, and dispersal distance.

\subsection{SCENARIO DEFINITION: NETWORK AND MATRIX CHARACTERISTICS}

We established three different scenarios (two static and one spatio-temporal) to represent the landscape (i.e., the combination of habitat network and matrix characteristics) for the different connectivity approaches. These scenarios encompassed: (a) the number, distribution, and area of focal habitat patches; (b) the characteristics of the surrounding matrix; (c) the spatial probability of connectivity between patches; and (d) the temporal probability of connectivity between spatio-temporal patches. These characteristics varied from $\mathrm{t} 1 \mathrm{to} \mathrm{t} 2$, and therefore each run of the static approach ( $\mathrm{s} 1$ and $\mathrm{s} 2$ ), as well as the spatio-temporal approach, had a different scenario associated. However, the time-varying approach did not require an additional scenario as it only considers together the two static approaches (i.e., scenarios).

\subsubsection{Definition of habitat patches and nodes}

Firstly, habitat cells were determined as the pixels covered by broadleaf forest at $\mathrm{t} 1$, $\mathrm{t} 2$, or both time snapshots according to CORINE land cover maps from 1990 and 2018. Subsequently, these habitat cells were classified as a) "stable" when identified as habitat at both time snapshots; b) "lost" if they were identified only at $\mathrm{t} 1$, and c) "gained" when identified only at $\mathrm{t} 2$. We then delineated habitat patches as contiguous habitat cells with the same temporal classification (stable, lost, or gained) with a surface over 50 ha. This big patch size threshold was set for two reasons: firstly, to ensure that patches can host individuals throughout their entire lifetime to overcome species connectivity over generations (Keeley et al. 2018) and secondly, for feasible computational processing. The resulting habitat patches defined the network nodes (Urban et al. 2009), which were qualified by their area (i.e., patch attribute). We used the whole set of nodes under the spatio-temporal scenario. However, we only used stable and lost nodes in the initial static scenario, while stable and gained nodes in the final static scenario (Fig. 3). 


\subsubsection{Landscape matrix characterization: Resistance surface}

Next, the landscape matrix of each scenario was characterized through resistance surfaces (Tischendorf and Fahrig 2000; Spear et al. 2010; Zeller et al. 2012). These surfaces depicted how the landscape allowed or impeded species movement. To create them, we assigned different resistance values to each land cover class/use according to Table S1 (in the supplementary data) based on previous studies of forest species in Spain (Gurrutxaga et al. 2011; Ruiz-González et al. 2014; de la Fuente et al. 2018) and expert opinion. These resistance values ranged from 1 to 1000, with increasing values corresponding to classes that restrain forest species movement. Resistance surfaces for the initial and final static scenarios were created from CORINE land cover maps at $\mathrm{t} 1$ and $\mathrm{t} 2$ respectively combined with the distribution of roads from OpenStreetMap (www.openstreetmap.org) to include the influence of linear infrastructures in species movements (Banfield et al. 2020). On the other hand, the resistance surface of the spatio-temporal scenario was defined as the mean of the two static surfaces. All resistance surfaces were processed at a spatial resolution of $200 \mathrm{~m}$.

\subsubsection{Connections between nodes: spatial probability of dispersal}

We applied least cost path modeling (Adriaensen et al. 2003) to determine the connections among nodes centroids with the R package gDistance. For each scenario, we connected each node with all the nodes of the same ecoregion. The effective distance of these connections (i.e., the accumulated resistance along the least cost path) depicted the difficulty of moving between each pair of nodes through the resistance surface. These effective distances were then converted into probabilities of direct spatial dispersal between each pair of nodes (Appendix S1). This conversion was calculated as the negative exponential function of the effective distance between patches (Saura and Pascual-Hortal 2007; Gurrutxaga et al. 2011; Saura et al. 2017, 2018). This function was adjusted to the dispersal abilities of the focal species by assigning a probability of 0.5 to the species median effective dispersal distance. To estimate the species median effective dispersal distance, we multiplied the previously defined lineal dispersal distances (i.e., 1, 2, 5, 10, 30, $50 \mathrm{~km}$ ) by the median landscape resistance value (Gurrutxaga and Saura 2013) of the spatio-temporal scenario. Finally, we excluded the connections with a spatial probability of connectivity below 0.001 and obtained a set of viable connections between nodes with their spatial probability of connection for each scenario and dispersal distance. We used R software for the calculations.

\subsubsection{Spatio-temporal adjustment of connections: temporal probability of dispersal}

In the spatio-temporal scenario, aside from the spatial probability of dispersal, we incorporated a temporal component (i.e., the temporal probability of dispersal between nodes) (Martensen et al. 2017). On the one hand, this temporal component (Table 2 and Fig. 3) dealt with the directionality of the 
network: successful direct spatio-temporal connectivity only occurred with the connection of patches active at an initial time 11 with patches active at a later final time t2. These links only existed in this forward direction, as it is impossible to move from future to past patches. Therefore, links that started at a gain node or ended at a loss node were associated with a null temporal probability of connectivity. On the other hand, the temporal component also depicted nodes coexistence: species could only move between contemporary patches that are active simultaneously at any intermediate time. In this respect, links starting and/or ending at stable nodes had an associated temporal probability of 1 , as the starting and ending nodes were sure to coexist during a certain period of time. Finally, links starting at a loss node and finishing at a gain node had an associated temporal probability of 0.5 , since their coexistence cannot be assured. However, we did not examine the length of the overlapping time period, which could modify this probability. To incorporate this temporal component in the spatio-temporal scenario we multiplied the spatial probability by the temporal probability of connectivity of each link. It resulted in the spatiotemporal probability that an individual moves from a given node at $\mathrm{t} 1$ to another node at $\mathrm{t} 2$.

Table 2

Temporal probability of connectivity from a source node at $\mathrm{t} 1$ (1990) to a destination node at t2 (2018).

\begin{tabular}{|lllll|}
\hline & & \multicolumn{3}{c|}{ Destination node } \\
\hline \multirow{3}{*}{ Source node } & Stable & Lost & Gained \\
\cline { 2 - 6 } & Stable & 1 & 0 & 1 \\
\cline { 2 - 6 } & Lost & 1 & 0 & 0.5 \\
\cline { 2 - 6 } & Gained & 0 & 0 & 0 \\
\hline
\end{tabular}

\subsection{CONNECTIVITY ANALYSES}

We performed a comparative assessment of the analyzed approaches (static, time-varying, and spatiotemporal) and the different ecoregions and dispersal distances. To do so, we calculated and contrasted the following connectivity analyses: i) the overall connectivity of the network (section 2.4.1), and ii) prioritization of key habitat patches to maintain the connectivity (section 2.4.2). To analyze connectivity, we used graph-based habitat availability metrics (Pascual-Hortal and Saura 2006; Saura and PascualHortal 2007; Saura and Rubio 2010), which integrate intrapatch connectivity (habitat available within habitat patches) with interpatch connectivity (habitat reachable through connections between patches). All connectivity metrics were calculated with the command line Conefor software (Saura and Torné 2009, www.conefor.org). The spatio-temporal scenario required the version for directed networks.

\subsubsection{Overall connectivity}


We used the Equivalent Connected Area (ECA, Saura et al. 2011; Santini et al. 2016) to determine the overall level of connectivity of each ecoregion, scenario, and dispersal distance. ECA (Table 3 ) is defined as the area of a single habitat patch that would provide the same amount of connectivity (probability of connectivity or PC, Table 3 ) as the actual pattern of habitat patches in the whole landscape. We calculated the ECA for the two static scenarios $\left(\mathrm{ECA}_{1}\right.$ at $\mathrm{t}_{1}$ and $E C \mathrm{~A}_{2}$ at $\left.\mathrm{t}_{2}\right)$, while for the spatio-temporal one we used a dynamic adaptation of this metric $\left(E C A_{s t}\right)$ (Martensen et al. 2017), best suited for the particularities of this scenario. The ECA of the entire study area was calculated as the sum of the resulting ECA value of each ecoregion. Afterward, we calculated the connectivity change (dECA, Table 3): (a) from the initial to the final static scenario (dECAtv) to measure the changes in connectivity over time (time-varying connectivity); and (b) from the initial to the spatio-temporal scenario (dECAst) to measure the difference in connectivity between following a static and a spatio-temporal approach. ECA and dECA metrics have area units and thus can be easily interpreted and compared with the existing focal habitat area, and its variation dA (Table 3) (Saura et al. 2011). 
Table 3

Metrics description. The metrics subindices represent the approach: 1 for the initial static approach in

1990; 2 for the final static approach in 2018; tv for the time-varying, and st for the spatio-temporal approach. For details on PC and ECA metrics see Saura 2007; Saura and Rubio 2010 and Martensen et al. 2017 for details on the spatio-temporal adaptation.

\begin{tabular}{|c|c|}
\hline Metric (units) & Description \\
\hline$A(h a)$ & Focal habitat area. Calculated at t1 $\left(\mathrm{A}_{1}\right)$ and t2 $\left(\mathrm{A}_{2}\right)$ \\
\hline$d A=\frac{A_{2}-A_{1}}{A_{1}} \cdot 100(\%)$ & $\begin{array}{l}\text { Focal habitat area change: Percentage of increase/decrease in habitat area } \\
\text { between } \mathrm{t} 2 \text { and } \mathrm{t} 1\end{array}$ \\
\hline$P C$ & $\begin{array}{l}\text { Probability of Connectivity: Probability that two randomly selected points within } \\
\text { the landscape fall into spatially connected habitat patches. Calculated at t } 1\left(\mathrm{PC}_{1}\right) \\
\text { and t2 }\left(\mathrm{PC}_{2}\right)\end{array}$ \\
\hline$P C_{s t}$ & $\begin{array}{l}\text { Spatio-temporal Probability of connectivity: Probability that two randomly } \\
\text { selected points within the landscape, one at } t 1 \text { and the other one at t } 2 \text {, fall into } \\
\text { spatially and temporally connected habitat patches. }\end{array}$ \\
\hline$d P C$ & $\begin{array}{l}\text { Relative importance of each node to overall connectivity. Calculated for every } \\
\text { approach: } \mathrm{dPC}_{1}, \mathrm{dPC}_{2}, \mathrm{dPC}_{\mathrm{tv}}, \text { and } \mathrm{dPC}_{\mathrm{st}} \text {. }\end{array}$ \\
\hline ECA (ha) & $\begin{array}{l}\text { Overall connectivity: Equivalent Connected area or area of a single habitat patch } \\
\text { that would provide the same PC as the actual pattern of habitat patches in the } \\
\text { whole landscape. }\end{array}$ \\
\hline$E C A_{s t}(h a)$ & $\begin{array}{l}\text { Spatio-temporal overall connectivity: the area of a single stable habitat patch that } \\
\text { would provide the same PCst as the network composed of the set of lost, gained, } \\
\text { and stable habitat nodes of the landscape }\end{array}$ \\
\hline$d E C A_{t_{v}}=\frac{E C A_{2}-E C A_{A_{1}}}{E C A_{A_{1}}} \cdot 100(\%)$ & $\begin{array}{l}\text { Connectivity change in the time-varying approach: Percentage of } \\
\text { increase/decrease in the connected habitat area from t } 2 \text { to } t 1\end{array}$ \\
\hline$d E C A_{s t}=\frac{E C A_{s e}-E C A_{1_{1}}}{E C A_{A_{1}}}, 100(\%)$ & $\begin{array}{l}\text { Connectivity change in the spatio-temporal approach: Percentage of } \\
\text { increase/decrease in the connected habitat area from the spatio-temporal to the } \\
\text { initial static scenario. }\end{array}$ \\
\hline
\end{tabular}

\subsubsection{Identification of priority habitat patches}

To characterize patches importance, we used the relative variation in the metric PC (dPC, Table 3 ) when systematically removing each habitat patch (Saura and Pascual-Hortal 2007). The patches with the highest $\mathrm{dPC}$ are the most important and irreplaceable to maintain the overall landscape connectivity, and therefore should be considered as critical and priority elements. To facilitate comparability between ecoregions and approaches, we integrated the patch importance of all dispersal distances considered (i.e., to account for the diversity of species associated with the focal habitat). For this purpose, we minmax normalized and summed the individual importance of all dispersal distances. We obtained a generalized relative importance for each patch and scenario $\left(\mathrm{dPC}_{1}, \mathrm{dPC}_{2}\right.$, and $\mathrm{dPC}_{\mathrm{st}}$ for the static at $\mathrm{t} 1$, static at $\mathrm{t} 2$, and spatio-temporal scenarios respectively). We used separately $\mathrm{dPC}_{1}$ and $\mathrm{dPC}_{2}$ to inform 
about patches importance in the two static approaches, however, we summed these two metrics to measure the time-varying connectivity importance $\left(\mathrm{dPC}_{\mathrm{tv}}\right)$.

Finally, to select the priority patches of each ecoregion and approach we identified the patches with the highest generalized relative importance whose area sum less than $10 \%$ of the total ecoregion area. To ensure comparability between the three connectivity approaches, we selected two sets of priority patches for each of the two dynamic approaches (time-varying and spatio-temporal), one including only patches active at $\mathrm{t} 1$, and another one with patches active at $\mathrm{t} 2$. In this way, six sets of priority patches were

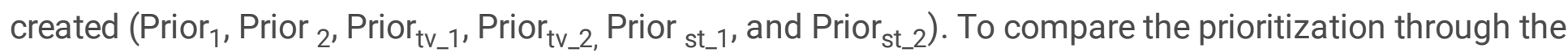
different connectivity approaches, we calculated the percentage of priority area shared by the six sets of priority patches. These percentages of shared priority area were calculated for each ecoregion and for the whole study area by summing the priority areas of every ecoregion.

\section{Results}

\subsection{Changes in the topology of the habitat network over time.}

There was a general increase in broadleaf forest area (dA) in the Iberian Peninsula of $40.11 \%$ (Table S2, Fig. 4), covering 11 and $15 \%$ of the study area in 1990 and 2018 respectively. Most of the habitat was classified as gained (absent at $\mathrm{t} 1$ and present at $\mathrm{t} 2$ ), followed by stable and lost (Fig. 4). Most ecoregions experienced an increase in the total habitat area and the mean patch size (Table S2). Only ecoregion 21 (Southwest Iberian Mediterranean sclerophyllous and mixed forests) showed a decrease in its total habitat area and mean patch size. The ecoregion 19 (Southeastern Iberian shrubs and woodlands) did not have any habitat patch in 1990 and only a small patch in 2018, therefore we did not present the results of this ecoregion. Inland Iberian ecoregions (Iberian sclerophyllous and semi-deciduous forests, and Iberian conifer forests ecoregions, i.e., ecoregions 9 and 8 ) had the smallest relative habitat area (i.e., the proportion of area covered by habitat) and the smallest mean habitat patch size at both time snapshots. Despite the total increase in habitat, the results also showed that there was a degradation or loss of quality of the surrounding landscape matrix for the movement of focal species: the mean resistance of the study area increased from 72.32 to 81.28 .

\subsection{Static connectivity}

As expected, the overall connectivity (ECA) increased with the dispersal abilities of the species (Fig. 5) at both $\mathrm{s} 1$ and $\mathrm{s} 2$ static scenarios. This growth in connectivity is more notable in low to medium dispersal distances (from 1 to $20 \mathrm{~km}$ ). Priority patches drastically differed between static scenarios (Table S3, Fig. s4). In fact, only $35.9 \%$ of the final priority patches (at t2) of the entire study area were identified as a

priority in the initial scenario (at $\mathrm{t} 1$ ). This percentage of shared priority habitat area between the two static scenarios varied between ecoregions from 0 to $60.7 \%$ in Cantabrian mixed forests (ecoregion 6), and Southwest Iberian Mediterranean sclerophyllous and mixed forests (ecoregion 21) respectively. 


\subsection{Time-varying connectivity}

The overall connectivity (ECA) showed a mean increase of 39.57\% (standard deviation of 1.2) from 1990 to 2018 for all dispersal distances (Fig. 5). This connectivity change $\left(\mathrm{dECA}_{\mathrm{tv}}\right)$ remained positive and stable across all dispersal distances, and with a very similar magnitude to the habitat area change (dA) when considering the whole study area (Fig. S1). However, most ecoregions showed a higher increase in overall connectivity for species with low dispersal capacity (Fig. S2), indicating that vagile species are less sensitive to changes in connectivity. In fact, in most ecoregions, this increase in overall connectivity was greater than the increase in total habitat area for short dispersal distances, while it was similar or smaller for large dispersal distances (Figure S3). On the contrary, ecoregions 6 (Cantabrian mixed forests), 8 (Iberian conifer forests), and 21 (Southwest Iberian Mediterranean sclerophyllous and mixed forests) showed a larger increase in habitat area than in overall connectivity. Particularly, this last ecoregion was the only region that experienced a decrease in habitat connectivity. The priority areas identified when following a time-varying approach covered a mean of 99.8 and $98.7 \%$ of the priority area from the initial and final static scenarios respectively (Table S3 and Fig. S4).

\subsection{Spatio-temporal connectivity}

The overall connectivity in the spatio-temporal scenario $\left(\mathrm{ECA}_{\mathrm{st}}\right)$ was in an intermediate state between the two static scenarios for all dispersal distances (Fig. 5). As in the static scenarios, the spatio-temporal overall connectivity increased with the dispersal distance for species with limited dispersal capabilities and remained almost stable for medium and large dispersal distances. Generally, there was a positive connectivity change $\left(\mathrm{dECA}_{\mathrm{st}}\right.$ ) (Fig. S1) which highlights the connectivity gain from t1 to t2. Most ecoregions also followed this trend (Fig. S5), yet ecoregions 8, 16, and 21 (Iberian conifer forests, Northwest Iberian montane forests, and Southwest Iberian Mediterranean sclerophyllous and mixed forests) had less connectivity in the spatio-temporal scenario than the initial static scenario, as shown by the negative connectivity increase. This last ecoregion experienced a loss of both habitat and connectivity, and thus, this negative change in connectivity was expected. However, ecoregions 8 and 16 presented an increase in habitat and connectivity from 1990 to 2018 when not considering the spatiotemporal dynamism.

Priority nodes identified in the spatio-temporal scenario matched closely with those from the static scenarios (Table S3 and Fig. S4 in the supplementary data). A mean of 98.9 and $97.9 \%$ of the priority patches in the initial and final static scenarios were also detected as priority areas in the spatio-temporal scenario. Therefore, the results of spatio-temporal connectivity identified priority conservation areas to ensure species range shifts, but also the maintenance of connectivity in specific snapshots in a varying landscape network. Additionally, $97 \%$ of the time-varying priority area was also identified as a priority when following the spatio-temporal approach. That is to say, the resulting key conservation areas were almost the same in the two approaches that account for the landscape dynamism.

\section{Discussion}


The abundant evidence of climate (Mora et al. 2013) and land-use changes (Song et al. 2018), and their influence on species distribution (Parmesan and Yohe 2003; Chen et al. 2011; Davidson et al. 2020) highlight the need to incorporate the landscape dynamism in conservation studies. Understanding how these landscape changes influence species connectivity is an important step forward in contemporary connectivity research and conservation planning. Habitat availability indices have been shown to be very useful for quantifying overall connectivity and identifying critical elements to maintain or enhance it. However, so far, they have been mainly used under a static approach (Dondina et al. 2017; de la Fuente et al. 2018; Cisneros-Araujo et al. 2021). Nevertheless, they have enormous potential to be adapted to different spatial and temporal probabilities of connectivity and deal with the directionality of the links. Therefore, they are a sound option to account for dynamism in connectivity analyses. This work aimed to gain insights into the use of these indices under two different dynamic approaches and to assess potential conceptual and practical differences among them and with traditional static approaches. Furthermore, the variety of considered ecoregions and species (i.e., dispersal abilities) allow a comprehensive interpretation of the results to advance in the incorporation of functional connectivity concerns for a wide array of species in changing landscapes. Therefore, this study may set a practical example to guide other analyses within a different management context (i.e., different study areas, focal species, landscape changes, and time snapshots). Particularly, the framework here exposed may be applied to future projections which would allow conservationists to anticipate future landscape changes and to implement improved measures with more functional and enduring conservation results.

\subsection{Changes in the topology of the habitat network over time}

We found a generalized increase in the broadleaf forest area in the Iberian Peninsula. This growth is consistent with other studies that found a net forest gain in the last years in Europe, and specifically in Spain and Portugal (FAO 2015), and all the biomes found in the Iberian Peninsula (Song et al. 2018), but especially in mountainous (ecoregions 33,16 , and 8 ) and temperate oceanic regions (ecoregion 6). However, this increase in forest area differs from other studies that found an overall forest loss in Spain and Portugal (Hansen et al. 2013). Nevertheless, all these previous studies examine general forest change rather than focusing only on broadleaf forests. Mixed or coniferous forest areas may have changed to broadleaf, appearing as habitat gain only in this study. Other possible causes of discrepancies might be the definition of forest area and the spatial and temporal resolution.

This broadleaf forest increase might be the result of recent agricultural land abandonment, afforestation activities, conservation policies, and the establishment of tree plantations. However, this net increase does not account for habitat quality. The gain of early successional or low heterogeneity forests may exceed the loss of mature and diverse forests, but not completely replace the ecosystem processes. In fact, many mature pure stands may be transformed into mono-species plantations (Teixido et al. 2010). These areas would be considered here as stable habitat patches besides the great loss of biodiversity and habitat quality. 


\subsection{Time-varying connectivity: Differences in connectivity between two snapshots}

The results confirm that connectivity changes over time as given by the varying value of overall connectivity (Fig. 5) and the different priority habitat patches between the two snapshots (Table S3 in supplementary material). These changes should be accounted for when planning optimal conservation measures to lengthen the beneficial effects of the measures. In this study, landscape changes in habitat amount and distribution and in the surrounding matrix resulted in a large improvement in connectivity in the period analyzed from 1990 to 2018 . There was a slightly larger increase in connectivity than in the total focal habitat area (Fig. S2). This might indicate that the habitat patches in 2018 were better connected because of the added habitat area per se (intrapatch connectivity) but also due to additional or improved connections between patches (interpatch connectivity). These improved connections might be the result of the gained habitat patches enhancing the connections with and between stable patches, or due to better characteristics of the intermediate matrix (even though there is an overall resistance increase, the matrix might have improved in-between the two connecting patches).

We found that landscape changes in most ecoregions had bigger impacts in connectivity for species with low dispersal distances (Fig. S2 in supplementary data): the gained or improved connections seem to be especially beneficial to short dispersers (Saura et al. 2011). Therefore, gained habitat patches may have been located close to the stable patches, acting as stepping stones and improving interpatch connectivity mostly for short dispersers. On the other hand, connectivity changes for vagile species were similar to the variation in habitat area. This might justify the focus of connectivity analyses in species with low dispersal abilities, which are more sensitive to landscape changes.

\subsection{Spatio-temporal connectivity: connectivity between different time snapshots}

Unlike static and time-varying approaches, spatio-temporal studies focus on connections between different snapshots. Following other approaches might lead to misjudging species connectivity to their future ranges in changing landscapes. The differing results obtained from static and spatio-temporal connectivity approaches suggest that static approaches fail to account for the connectivity between snapshots. The overall connectivity according to the spatio-temporal approach was in between that of the two static approaches, being in this case study generally smaller in 1990 and larger in 2018. Thus, the landscape changes (in the habitat patches distribution and area and the surrounding matrix) led to a large final static connectivity that did not especially favor the spatio-temporal connectivity between the two time snapshots (Fig. 2). The resulting final static overall connectivity tended therefore to overestimate spatio-temporal connectivity. However, when there was habitat loss (ecoregion 21, Southwest Iberian Mediterranean sclerophyllous and mixed forests) the use of static approaches underestimated the connectivity between snapshots. The same pattern of over and underestimation with net habitat gain and loss was found in other studies (Martensen et al. 2017; Huang et al. 2020). 
The connectivity difference between the initial static and the spatio-temporal approaches decreased with the species dispersal distance (Figs. S1 and S5): species with low dispersal distances were more benefited from the spatio-temporal perspective than vagile species. This pattern was also found in other spatio-temporal connectivity studies (Martensen et al. 2017) and when comparing time-varying and static approaches (Fig. S2). This trend was particularly noticeable in Northwest Iberian montane forests (ecoregion 16), which even presented a loss in spatio-temporal connectivity for large dispersal distances (Fig. S5) despite the increase in habitat area (Table S2). This may be caused by the loss of links or stepping stones that connected distant nodes, which hindered the movement of long-distance dispersers. At the same time, the new habitat might have appeared close to other habitat patches already reachable for good dispersers and only enhancing the movement of species with low dispersal capacities.

When incorporating the temporal component to the probability of dispersal between patches Martensen et al. 2017 considered the auxiliary links aside from the direct links here accounted. These auxiliary links do not provide spatio-temporal connectivity by themselves as they do not connect nodes active at t1 with nodes active at $t 2$ (i.e., auxiliary links start at a gained node or finish at a lost node). However, they connect nodes that could coexist in time. The connection of these auxiliary links with other links could result in actual spatio-temporal connectivity. We did not consider this kind of link in our study, as their inclusion did not change the results and highly increased computing times.

\subsection{Patch prioritization}

The results show substantial differences in habitat prioritization between the two static approaches (Table S3 and Fig. S4). The key areas that maintain connectivity among contemporary patches largely varied from the initial to the final snapshots. Therefore, relying on traditional static connectivity models may lead to the prioritization of temporal suitable areas that would not persist under this condition for a long period. This finding further strengthens the idea of connectivity as a time-varying phenomenon (Saura et al. 2011; Beltrán et al. 2014; Bishop-Taylor et al. 2018b; Jennings et al. 2020).

The two dynamic approaches adopted here (i.e., time-varying and spatio-temporal) promote connectivity between coexisting patches at several snapshots and from past to future patches. Even though the two approaches accounted for landscape dynamism differently, they identified almost the same priority patches (Table S3, Fig. S4). That is to say, the patches with the highest long-term importance also contributed to a larger extent to species movements to their future ranges. The two dynamic approaches also captured neatly the priority areas from static scenarios at the two snapshots. Dynamic prioritization approaches are therefore useful to identify the key patches to promote connectivity at specific time snapshots, but they give extra consideration to patches critical at other time snapshots or with important spatio-temporal connections. However, only the spatio-temporal connectivity approach addresses all these issues running a unique model that remains useful despite changes in the landscape, while the time-varying prioritization approach requires running one model per time snapshot considered.

\section{Conclusions}


This study emphasizes the importance of considering the dynamism of changing landscapes in connectivity studies. Traditional static approaches did not accurately assess the impacts of these changes. We have seen two different ways to incorporate the dynamism in connectivity studies: a) by acknowledging that connectivity is a time-varying phenomenon and therefore, procuring long-term conservation measures; and $b$ ) by enhancing species movements from their past to future ranges. We have shown that following different connectivity approaches resulted in very different overall connectivity values. Therefore, it is important to select the appropriate approach depending on the study objectives and the dynamic perspective considered. Otherwise, we might over or underestimate the connectivity levels in and between different time snapshots. On the other hand, habitat patches prioritization by means of the two dynamic perspectives showed very similar results. Both of them also identified most of the priority areas from static approaches at the two snapshots considered. Therefore, conservation strategies based on the two dynamic prioritization approaches may equally deal with the dynamism of landscapes and still enhance traditional static connectivity. That is to say, following either approach to incorporate the dynamism would foster endurable conservation measures that enhance connectivity between contemporaneous habitat patches, but also species range shifts in time. However, further research in this direction is needed to draw conclusions in other study areas, landscape changes, and time windows.

\section{Declarations}

\section{Funding:}

This study was supported by funding from Universidad Politécnica de Madrid.

\section{Competing interests}

The authors declare that they have no competing interests

\section{Ethics approval and consent to participate}

Not applicable. However, authors comply with the IUCN Policy Statement on Research Involving Species at Risk of Extinction.

\section{Consent to participate}

Not applicable.

\section{Consent for publication}

Not applicable.

\section{Availability of data and materials}


The data that support the findings of this study are openly available at https://land.copernicus.eu/paneuropean/corine-land-cover.

\section{Code availability}

The scripts used to generate the analyses presented are available in "figshare" at https://figshare.com/s/b9411312d964895deac3, DOI 10.6084/m9.figshare.16799503 .

\section{Authors contribution}

All authors conceived the ideas and designed the methodology; TG analyzed the data and ran the connectivity models with help from MCM. TG led the writing of the manuscript and MCM reviewed it thoroughly. Both authors contributed critically to the drafts and gave final approval for publication.

\section{References}

1. Acevedo MA, Fletcher RJ, Tremblay RL, Meléndez-Ackerman EJ (2015) Spatial asymmetries in connectivity influence colonization-extinction dynamics. Oecologia 179:415-424. https://doi.org/10.1007/s00442-015-3361-z

2. Adriaensen F, Chardon JP, De Blust G et al (2003) The application of "least-cost" modelling as a functional landscape model. Landsc Urban Plan 64:233-247. https://doi.org/10.1016/S01692046(02)00242-6

3. Albert CH, Rayfield B, Dumitru M, Gonzalez A (2017) Applying network theory to prioritize multispecies habitat networks that are robust to climate and land-use change. Conserv Biol 31:1383-1396. https://doi.org/10.1111/cobi.12943

4. Banfield JE, Ciuti S, Nielsen CC, Boyce MS (2020) Cougar roadside habitat selection: Incorporating topography and traffic. Glob Ecol Conserv 23:1-11. https://doi.org/10.1016/j.gecco.2020.e01186

5. Beltrán BJ, Franklin J, Syphard AD et al (2014) Effects of climate change and urban development on the distribution and conservation of vegetation in a Mediterranean type ecosystem. Int J Geogr Inf Sci 28:1561-1589. https://doi.org/10.1080/13658816.2013.846472

6. Bishop-Taylor R, Tulbure MG, Broich M (2018a) Impact of hydroclimatic variability on regional-scale landscape connectivity across a dynamic dryland region. Ecol Indic 94:142-150. https://doi.org/10.1016/j.ecolind.2017.07.029

7. Bishop-Taylor R, Tulbure MG, Broich M (2018b) Evaluating static and dynamic landscape connectivity modelling using a 25-year remote sensing time series. Landsc Ecol 33:625-640. https://doi.org/10.1007/s10980-018-0624-1

8. Carroll C, Parks SA, Dobrowski SZ, Roberts DR (2018) Climatic, topographic, and anthropogenic factors determine connectivity between current and future climate analogs in North America. Glob Chang Biol 24:5318-5331. https://doi.org/10.1111/gcb.14373 
9. Chen IC, Hill JK, Ohlemüller R et al (2011) Rapid range shifts of species associated with high levels of climate warming. Science (80-) 333:1024-1026. https://doi.org/10.1126/science.1206432

10. Cisneros-Araujo P, Goicolea T, Mateo-Sánchez MC et al (2021) The role of remote sensing data in habitat suitability and connectivity modeling: Insights from the cantabrian brown bear. Remote Sens 13. https://doi.org/10.3390/rs13061138

11. Collinge SK (1996) Ecological consequences of habitat fragmentation: implications for landscape architecture and planning. Landsc Ecol 36:59-77

12. Conlisk E, Haeuser E, Flint A et al (2021) Pairing functional connectivity with population dynamics to prioritize corridors for Southern California spotted owls. Divers Distrib 1-13.

https://doi.org/10.1111/ddi.13235

13. Correa Ayram CA, Mendoza ME, Etter A, Pérez Salicrup DR (2015) Habitat connectivity in biodiversity conservation: A review of recent studies and applications. Prog Phys Geogr 40:1-32. https://doi.org/10.1177/0309133315598713

14. Costanza JK, Watling J, Sutherland R et al (2020) Preserving connectivity under climate and land-use change: No one-size-fits-all approach for focal species in similar habitats. Biol Conserv 248. https://doi.org/10.1016/j.biocon.2020.108678

15. Davidson SC, Bohrer G, Gurarie E et al (2020) Ecological insights from three decades of animal movement tracking across a changing Arctic. Science (80-) 370:712-715. https://doi.org/10.1126/science.abb7080 Ecological

16. de la Fuente B, Mateo-Sánchez MC, Rodríguez G et al (2018) Natura 2000 sites, public forests and riparian corridors: The connectivity backbone of forest green infrastructure. Land use policy 75:429441. https://doi.org/10.1016/j.landusepol.2018.04.002

17. Dondina O, Orioli V, Colli L et al (2017) Ecological network design from occurrence data by simulating species perception of the landscape. Landsc Ecol 33:275-287. https://doi.org/10.1007/s10980-0170600-1

18. Elsen PR, Monahan WB, Dougherty ER, Merenlender AM (2020) Keeping pace with climate change in global terrestrial protected areas. Sci Adv 6. https://doi.org/10.1126/sciadv.aay0814

19. Gastón A, Blázquez-Cabrera S, Ciudad C et al (2019) The role of forest canopy cover in habitat selection: insights from the Iberian lynx. Eur J Wildl Res 65:1-10. https://doi.org/10.1007/s10344019-1266-6

20. Gray M, Micheli E, Comendant T, Merenlender A (2020) Climate-wise habitat connectivity takes sustained stakeholder engagement. Land 9:1-21. https://doi.org/10.3390/land9110413

21. Gurrutxaga M, Rubio L, Saura S (2011) Landscape and Urban Planning Key connectors in protected forest area networks and the impact of highways: A transnational case study from the Cantabrian Range to the Western Alps (SW Europe). Landsc Urban Plan 101:310-320. https://doi.org/10.1016/j.landurbplan.2011.02.036

22. Gurrutxaga M, Saura S (2013) Prioritizing highway defragmentation locations for restoring landscape connectivity. Environ Conserv 41:157-164. https://doi.org/10.1017/S0376892913000325 
23. Hansen MC, Potapov PV, Moore R et al (2013) High-Resolution Global Maps of 21st-Century Forest Cover Change. Science (80-) 342:850-853. https://doi.org/10.1126/science.1244693

24. Honnay O, Verheyen K, Butaye $\mathrm{J}$ et al (2002) Possible effects of habitat fragmentation and climate change on the range of forest plant species. Ecol Lett 5:525-530. https://doi.org/10.1046/j.14610248.2002.00346.x

25. Huang JL, Andrello M, Martensen AC et al (2020) Importance of spatio-temporal connectivity to maintain species experiencing range shifts. Ecography (Cop) 43:591-603. https://doi.org/10.1111/ecog.04716

26. Jennings MK, Haeuser E, Foote D et al (2020) Planning for Dynamic Connectivity: Operationalizing Robust Decision-Making and Prioritization Across Landscapes Experiencing Climate and Land-Use Change. Land 9:341. https://doi.org/10.3390/land9100341

27. Keeley ATH, Ackerly DD, Basson G et al (2018a) LANDSCAPE CONNECTIVITY:WHY, HOW, AND WHAT NEXT

28. Keeley ATH, Ackerly DD, Cameron DR et al (2018b) New concepts, models, and assessments of climate-wise connectivity New concepts, models, and assessments of climate-wise connectivity. Environ Res Lett 13:1-18

29. Keeley ATH, Beier P, Gagnon JW (2016) Estimating landscape resistance from habitat suitability: effects of data source and nonlinearities. Landsc Ecol 31:2151-2162. https://doi.org/10.1007/s10980-016-0387-5

30. Keeley ATH, Beier P, Jenness JS (2021) Connectivity metrics for conservation planning and monitoring. Biol Conserv 255. https://doi.org/10.1016/j.biocon.2021.109008

31. Littlefield CE, McRae BH, Michalak JL et al (2017) Connecting today's climates to future climate analogs to facilitate movement of species under climate change. Conserv Biol 31:1397-1408. https://doi.org/10.1111/cobi.12938

32. Loarie SR, Duffy PB, Hamilton H et al (2009) The velocity of climate change. Nature 462:1052-1055. https://doi.org/10.1038/nature08649

33. Martensen AC, Saura S, Fortin MJ (2017) Spatio-temporal connectivity: assessing the amount of reachable habitat in dynamic landscapes. Methods Ecol Evol 8:1253-1264. https://doi.org/10.1111/2041-210X.12799

34. Mateo-Sánchez MC, Balkenhol N, Cushman S et al (2015) A comparative framework to infer landscape effects on population genetic structure: are habitat suitability models effective in explaining gene flow? Landsc Ecol 30:1405-1420. https://doi.org/10.1007/s10980-015-0194-4

35. Mateo Sánchez MC, Cushman SA, Saura S (2014) Scale dependence in habitat selection: the case of the endangered brown bear (Ursus arctos) in the Cantabrian Range (NW Spain). Int J Geogr Inf Sci 28:1531-1546. https://doi.org/10.1080/13658816.2013.776684

36. Mora C, Frazier AG, Longman RJ et al (2013) The projected timing of climate departure from recent variability. Nature 502:183-187. https://doi.org/10.1038/nature12540 
37. Nuñez TA, Lawler JJ, McRae BH et al (2013) Connectivity Planning to Address Climate Change. Conserv Biol 27:407-416. https://doi.org/10.1111/cobi.12014

38. Olson DM, Dinerstein E, Wikramanayake ED et al (2001) Terrestrial ecoregions of the world: a new map of life on Earth. Bioscience 51:933-938

39. Parmesan C, Yohe G (2003) A globally coherent fingerprint of climate change impacts across natural systems. Nature 421:37-42. https://doi.org/10.1038/nature01286

40. Pascual-Hortal L, Saura S (2006) Comparison and development of new graph-based landscape connectivity indices: towards the priorization of habitat patches and corridors for conservation. Landsc Ecol 21:959-967. https://doi.org/10.1007/s10980-006-0013-z

41. Poli C, Hightower J, Fletcher RJ (2019) Validating network connectivity with observed movement in experimental landscapes undergoing habitat destruction. J Appl Ecol 1-12. https://doi.org/10.1111/1365-2664.13624

42. Román-Palacios C, Wiens JJ (2020) Recent responses to climate change reveal the drivers of species extinction and survival. https://doi.org/10.1073/pnas.1913007117. PNAS

43. Root TL, Price JT, Hall KR et al (2003) Fingerprints of global warming on wild animals and plants. Nature 421:57-60. https://doi.org/10.1038/nature01333

44. Ruiz-González A, Gurrutxaga M, Cushman SA et al (2014) Landscape genetics for the empirical assessment of resistance surfaces: The European pine marten (Martes martes) as a target-species of a regional ecological network. PLoS ONE 9. https://doi.org/10.1371/journal.pone.0110552

45. Santini L, Di Marco M, Visconti P et al (2013) Ecological correlates of dispersal distance in terrestrial mammals. Hystrix. Ital J Mammal Online 1825-5272. https://doi.org/10.4404/hystrix-24.2-8746

46. Santini L, Saura S, Rondinini C (2016) Connectivity of the global network of protected areas. Divers Distrib 22:199-211. https://doi.org/10.1111/ddi.12390

47. Saura S (2007) A new habitat availability index to integrate connectivity in landscape conservation planning: Comparison with existing indices and application to a case study. 83:91-103. https://doi.org/10.1016/j.landurbplan.2007.03.005

48. Saura S, Bastin L, Battistella L et al (2017) Protected areas in the world 's ecoregions: How well connected are they ? Ecol Indic 76:144-158. https://doi.org/10.1016/j.ecolind.2016.12.047

49. Saura S, Bertzky B, Bastin L et al (2018) Protected area connectivity: Shortfalls in global targets and country-level priorities. Biol Conserv 219:53-67. https://doi.org/10.1016/j.biocon.2017.12.020

50. Saura S, Estreguil C, Mouton C, Rodríguez-Freire M (2011) Network analysis to assess landscape connectivity trends: Application to European forests (1990-2000). Ecol Indic 11:407-416. https://doi.org/10.1016/j.ecolind.2010.06.011

51. Saura S, Pascual-Hortal L (2007) A new habitat availability index to integrate connectivity in landscape conservation planning: Comparison with existing indices and application to a case study. Landsc Urban Plan 83:91-103. https://doi.org/10.1016/j.landurbplan.2007.03.005 
52. Saura S, Rubio L (2010) A common currency for the different ways in which patches and links can contribute to habitat availability and connectivity in the landscape. Ecography (Cop) 33:523-537. https://doi.org/10.1111/j.1600-0587.2009.05760.x

53. Saura S, Torné J (2009) Conefor Sensinode 2.2: A software package for quantifying the importance of habitat patches for landscape connectivity. Environ Model Softw 24:135-139. https://doi.org/10.1016/j.envsoft.2008.05.005

54. Song X, Hansen MC, Stehman SV et al (2018) Global land change 1982-2016. Nature 560:639-643. https://doi.org/10.1038/s41586-018-0411-9.Global

55. Spear SF, Balkenhol N, Fortin MJ et al (2010) Use of resistance surfaces for landscape genetic studies: considerations for parameterization and analysis. Mol Ecol 19:3576-3591. https://doi.org/10.1111/j.1365-294X.2010.04657.x

56. Sutherland GD, Harestad AS, Price K, Lertzman KP (2000) Scaling of natal dispersal distances in terrestrial birds and mammals. Ecol Soc 4. https://doi.org/10.5751/es-00184-040116

57. Taylor RG, Scanlon B, Döll P et al (2013) Groundwater and climate change: recent advances and a look forward. Nat Clim Chang 3:322-329. https://doi.org/10.1038/nclimate1744

58. Teixido AL, Quintanilla LG, Carreño F, Gutiérrez D (2010) Impacts of changes in land use and fragmentation patterns on Atlantic coastal forests in northern Spain. J Environ Manage 91:879-886. https://doi.org/10.1016/j.jenvman.2009.11.004

59. Tischendorf L, Fahrig L (2000) On the usage and measurement of landscape connectivity. Oikos $1: 7-19$

60. Urban DL, Minor ES, Treml EA, Robert S (2009) Graph models of habitat mosaics. Ecol Lett 12:260273. https://doi.org/10.1111/j.1461-0248.2008.01271.x

61. Zeller KA, Lewsion R, Fletcher RJ et al (2020) Understanding the Importance of Dynamic Landscape Connectivity. Land 9:1-15. https://doi.org/10.1093/oso/9780198838388.003.0005

62. Zeller KA, McGarigal K, Whiteley AR (2012) Estimating landscape resistance to movement: A review. Landsc Ecol 27:777-797. https://doi.org/10.1007/s10980-012-9737-0

63. Zhao H, Liu D, Li F et al (2021) Incorporating spatio-temporal connectivity for prioritized conservation of individual habitat patches in a dynamic landscape. Ecol Indic 124:107414. https://doi.org/10.1016/j.ecolind.2021.107414

\section{Figures}




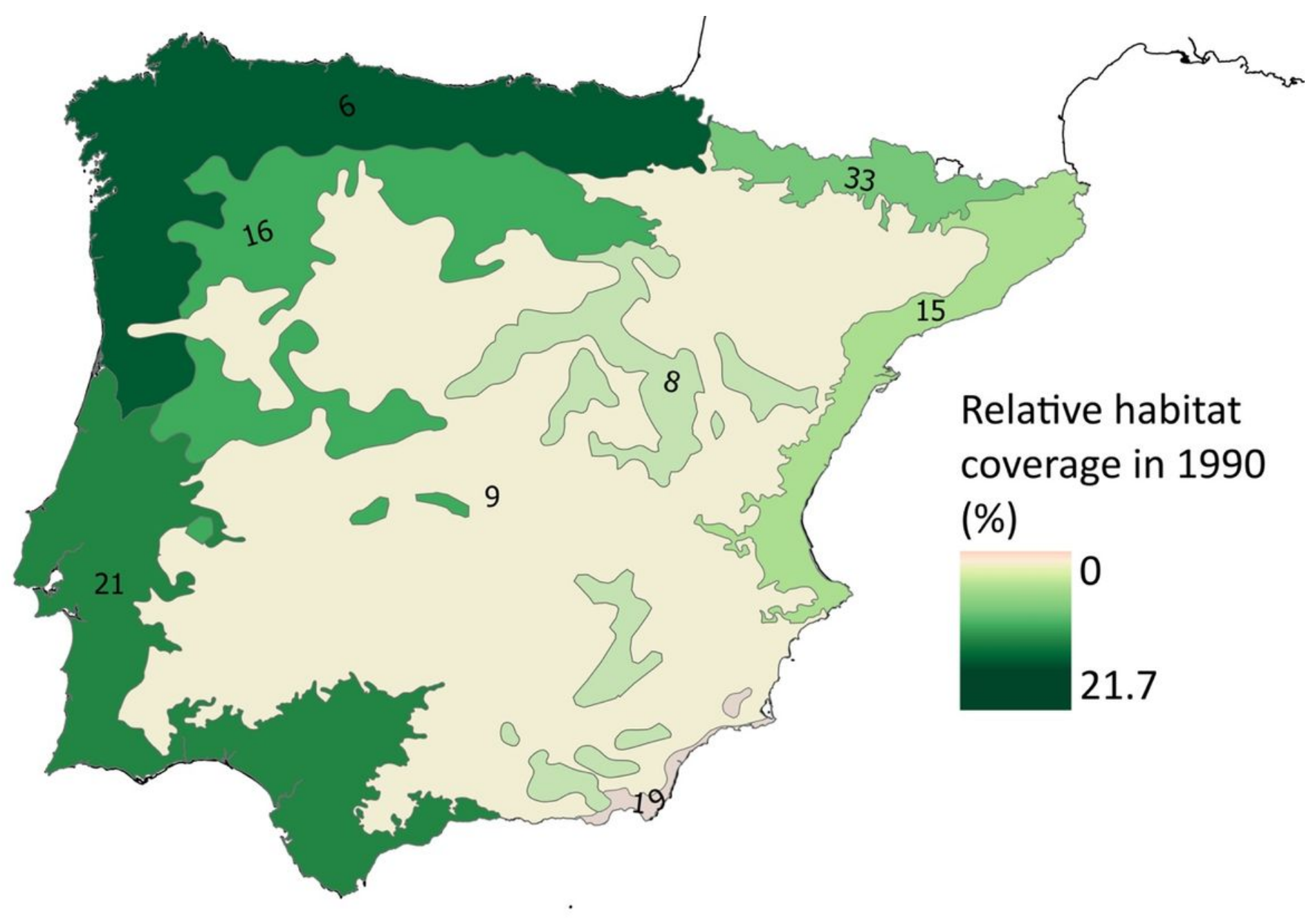

Figure 1

Ecoregions of the Iberian Peninsula and their relative habitat coverage (i.e., percentage of ecoregion area covered by broadleaf forest) in 1990. Ecoregions names can be found in Table 1. 
1 Scenario definition: Network and matrix characteristics

A. Definition of habitat patches

B. Resistance surface

C. Connections between patches

D. Temporal probability of connections

\section{Connectivity analyses}

A. Overall connectivity

B. Identification of priority habitat patches

\section{Three scenarios:}

Static at t1

Static at t 2

Spatio-temporal

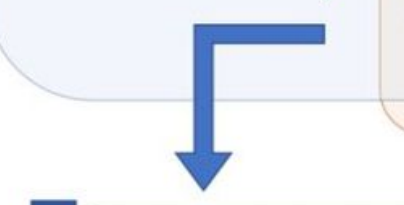

3 Comparison of the approaches results

\begin{tabular}{|c|c|c|c|c|}
\hline \multirow{2}{*}{$\begin{array}{l}\text { Comparison of the } \\
\text { approaches result }\end{array}$} & \multirow[b]{2}{*}{ Approach } & \multicolumn{2}{|c|}{ Overall connectivity } & \multirow{2}{*}{ Priority patche } \\
\hline & & Connectivity level & Connectivity change & \\
\hline \multirow{2}{*}{ Static } & Static at $\mathrm{t} 1$ & $\mathrm{ECA}_{1}$ & - & Prior $_{1}$ \\
\hline & Static at $\mathrm{t} 2$ & $\mathrm{ECA}_{2}$ & - & Prior $_{2}$ \\
\hline \multirow{2}{*}{ Dynamic } & Time-varying & - & $\mathrm{dECA}_{\mathrm{tv}}$ & Prior $_{t_{1} \_}$, Prior $_{\mathrm{tv}_{-}}$ \\
\hline & Spatio-temporal & $\mathrm{ECA}_{\mathrm{st}}$ & $\mathrm{dECA}_{\mathrm{st}}$ & Prior $_{\text {st_1 }}$, Prior $_{\text {st_2 }}$ \\
\hline
\end{tabular}

\section{Figure 2}

Methodological workflow scheme. 1. Scenario definition (section 2.3): We developed three scenarios, i.e., the combination of network and matrix characteristics: static at t1 (1990), static at t2 (2018), and spatiotemporal from $\mathrm{t} 1$ to $\mathrm{t} 2$. To do so, we (a) identified the habitat patches, (b) calculated the resistance surface, (c) determined the connections between patches with least cost path modeling and the spatial probability of connection, and (d) calculated the temporal probability of the connections. This was done separately for the three scenarios. 2. Connectivity analyses (section 2.4): we calculated different connectivity metrics (Table 3) to measure: (a) the overall connectivity and (b) the individual patch importance to ultimately identify priority conservation patches. This second stage was conducted for the three scenarios and six dispersal distances. 3. Comparison of connectivity approaches: We compared the connectivity results when following a static, time-varying, or spatio-temporal approach. 

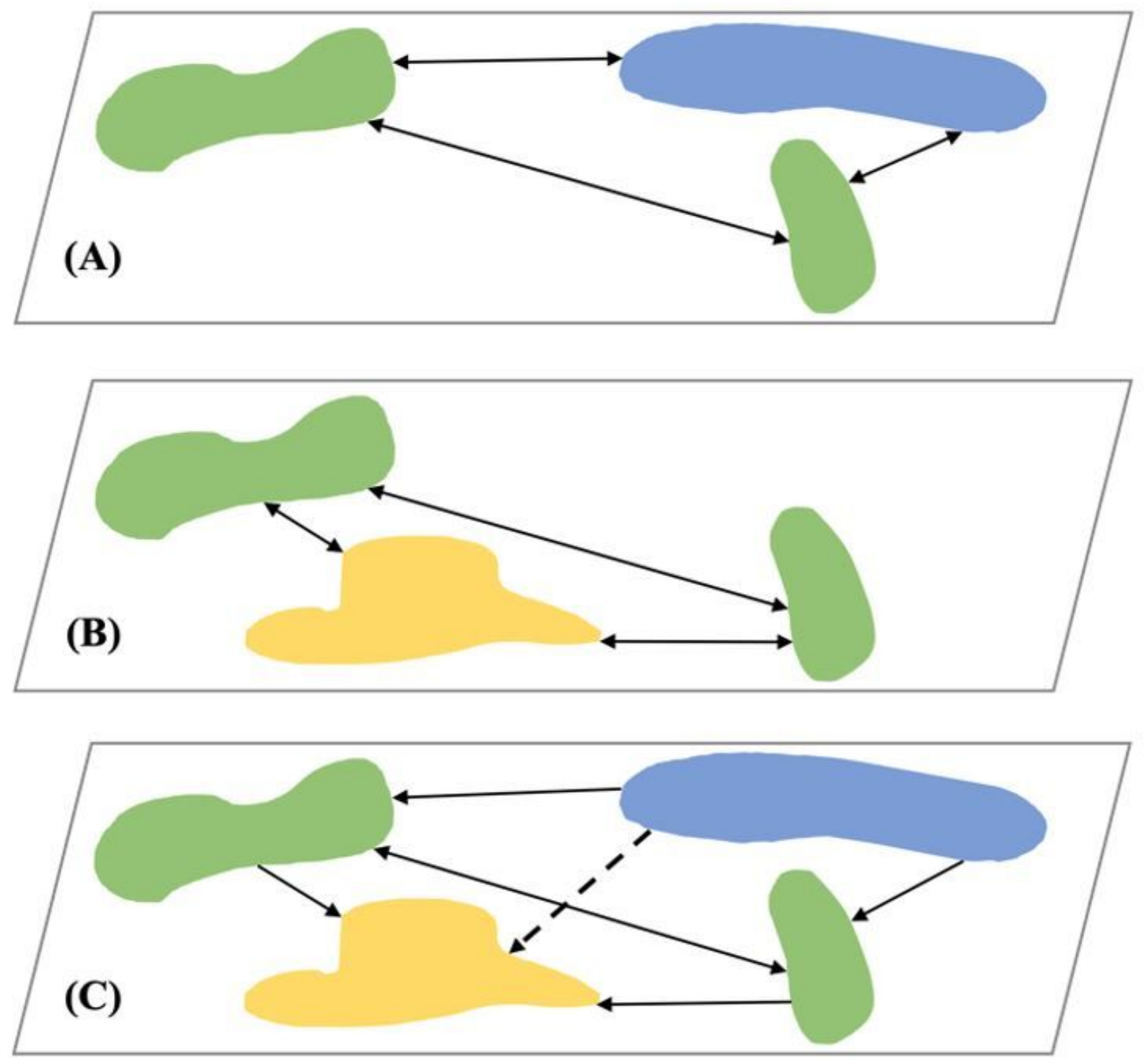

\section{Figure 3}

Representation of the habitat patches and connections between them for the three scenarios: (A) static at t1 (1990); (B) static at t2 (2018); and (C) spatio-temporal from t1 to t2. Stable (active at t1 and t2), lost (active at $\mathrm{t} 1$ ), and gained (active at $\mathrm{t} 2$ ) patches are represented as green, blue, and yellow shapes respectively. One and two-way arrows represent the possible links and the direction of the connection between nodes. All the connections in the static scenarios (A and B) are bidirectional, while they are directed in the spatio-temporal scenario (C), only connecting nodes active at t1 (stable and lost) with nodes active at $\mathrm{t} 2$ (stable and gained). Solid and dashed arrows in the spatio-temporal scenario denote the temporal probability nodes coexistence of 1 and 0.5 respectively. 


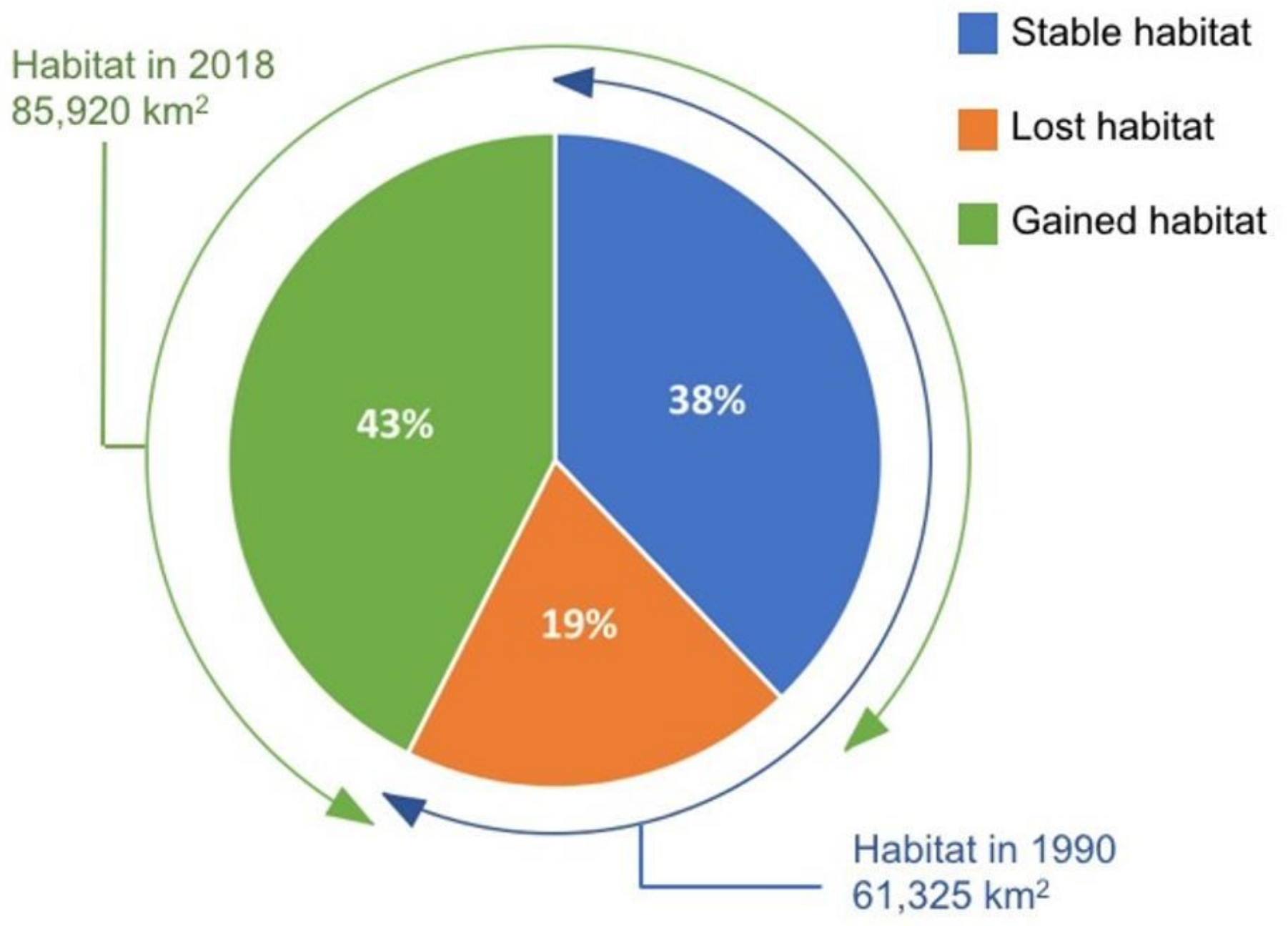

Figure 4

Proportion of each habitat class (stable, lost, and gained) in the Iberian Peninsula, and total habitat (deciduous forest) area in 1990 and 2018. 
$\rightarrow \mathrm{A}_{1} \rightarrow \mathrm{A}_{2} \rightarrow \mathrm{ECA}_{1} \rightarrow \mathrm{ECA}_{2} \rightarrow \mathrm{ECA}_{\mathrm{st}}$

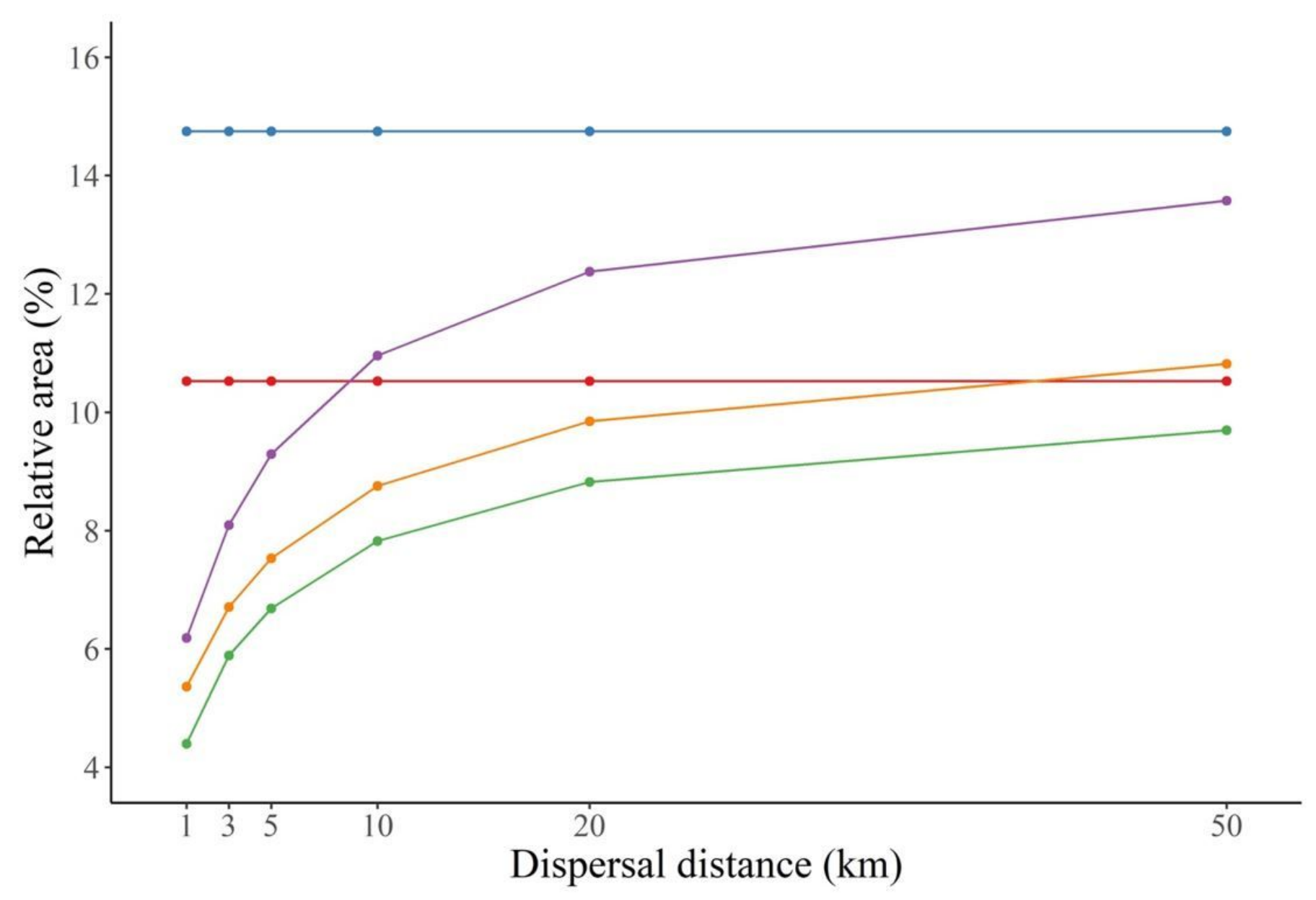

Figure 5

Relative habitat area and overall connectivity: Percentage of study area covered by focal habitat (A) and by connected habitat (ECA) in the initial static (in 1990, subscript 1), final static (in 2018, subscript 2), and spatio-temporal (subscript st) scenarios.

\section{Supplementary Files}

This is a list of supplementary files associated with this preprint. Click to download.

- DUSsuppmat111021.docx 